\title{
A SURVEY ON STOCK PRICE PREDICTION USING MACHINE LEARNING
}

\author{
Manavi Mishra, Manjushree Patil, Geetanjali Raut \\ Student, Department of EXTC \\ St. John College of Engineering, Palghar \\ Maharashtra, India \\ Tushar Chaudhari \\ Project Guide, Asst. Professor \\ Department of EXTC \\ St. John College of Engineering, Palghar \\ Maharashtra, India
}

\begin{abstract}
Stock returns are very fluctuating in nature. They rely upon various factors like previous stock prices, current market trends, financial news, etc. To feature their annual income, people have now started watching stock investments as a remunerative option. There are many tools available to investors using technical analysis to form decisions. With expert guidance and intelligent planning, we will almost double our annual income through stock returns. These days, social media has become a mirror. It reflects people's thoughts and opinions on any particular event or news. Sentiments of the general public associated with an organization can have an upshot on its stock prices. This paper surveys various machine learning techniques and algorithms employed to boost the accuracy of stock price prediction.
\end{abstract}

Keywords - Stock prices, Technical analysis, Stock Returns, Social Media, Sentiments

\section{INTRODUCTION}

Stock price prediction is one of the foremost important problems faced by many investors. This increases the danger of investing our earned money with the fear of losing it. Hence, many are scared to speculate within the stock exchange. Investing within the Stock markets generates significant revenue for the country. If a successful model for stock prediction is developed, we will gain insight into the market movement over time, spotting the trends that might otherwise not have been observed. Together with the increasing computational power of computers, machine learning is going to be a coherent method to resolve this problem. Our main motivation for this project is to develop a much better stock price prediction system that the investments increases and therefore the investors can maximize their profits.

\section{LITERAUTRE SURVEY}

\section{Stock Market Prediction using Machine Learning Algorithms: A Classification Study}

The objective of this paper is to classify different machine learning algorithms. This paper introduced the concept of economic derivatives just like the "no arbitrage" principle and people of the predictive model, like stochastic process theory and efficient market hypothesis (EMH). News articles were analyzed and also the prediction was made on their basis. Steps involved in prediction were data preparation, analysis, aggregation, and visualization. The results were neutral, positive, and negative. Data were collected daily and predictions were made on Logistic Regression. The accuracy obtained was $70 \%$. A generalized linear model (LM) (binomial family) is often used as a logistic regression model. [1]

\section{Stock Market Prediction using Machine Learning Techniques}

The paper predicts the market performance of the Karachi stock market (KSE) using different machine learning techniques. Various methodologies were discussed like Single Layer Perceptron, Multi-Layer Perceptron, Radial Basis Function, and Support Vector Machines. The technique proposed is to use various factors impacting the market as input attributes for the machine learning model. Many different factors were found to own a major impact on the market performance. [2]

\section{Survey of Stock Market Prediction Using Machine Learning Approach}

This paper has proposed the survey of a widely known efficient regression approach to predict the stock exchange price from the stock exchange database. Various regression techniques like polynomial regression, rectilinear regression, RBF regression, and sigmoid regression were described. Polynomial regression is that the variety of statistical regression which is that the best fit exchange prediction because it is largely for the non-linear relationship between one dependent and another experimental variable. [3] 


\section{International Journal of Engineering Applied Sciences and Technology, 2021 \\ Vol. 5, Issue 11, ISSN No. 2455-2143, Pages 206-210 \\ Published Online March 2021 in IJEAST (http://www.ijeast.com)}

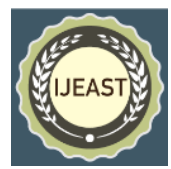

\section{Stock Market Prediction using Data Mining Techniques}

In this paper, the prediction of the exchange trends was done using machine learning models just like the Random Forest model and Support Vector Machine. The historical data, further as sentimental data, were analyzed as they players an important role in market performance. Researchers applied sentiment analysis on Twitter feeds to find the interrelationship among "public sentiment" and therefore the "market sentiment". Data retrieved from Twitter was wont to predict the general public mood. A Self Organizing Fuzzy Neural Network was used on predicted mood from the Twitter feeds and Dow-Jones Industrial Average values from the previous day to predict the movement of the securities market for the longer term. [4]

\section{Stock Closing Price Prediction Using Machine Learning}

This paper predicted the price of the stocks within the stock market of Thailand (SET). Various methodologies wont to predict the damage of the stock were the Multi-Layer Perceptron model, Support Vector Machine model, and Partial Least Square Classifier. The experimental result proves that Partial Least Square is that the best algorithm of the three algorithms to predict the stock terms. [5]

\section{Empirical Study on Stock Market Prediction Using Machine Learning}

This paper aims to review the securities market prediction using multiple Traditional, Machine learning, and Deep learning algorithms. together with these algorithms, the survey has also focused on various datasets used for securities market prediction, the features of those datasets were selected as input parameters, and also the evaluation metrics used for comparing the results of predictions. All of these were taken into consideration to create an efficient prediction. [6]

\section{DP-LSTM: Differential Privacy-inspired LSTM for Stock Prediction Using Financial News}

This paper proposes a novel profound deep neural network DPLSTM for stock value prediction. It coordinates news stories as restricted data and joins distinctive news sources through the differential security system. In view of the autoregressive moving average model (ARIMA), an estimation ARIMA is assembled by contemplating the data of monetary news stories in the model. At that point, an LSTM-based profound neural organization is outlined. It has three parts: LSTM, VADER model, and differential protection (DP) component. The planned DP-LSTM plan can altogether decrease forecast blemishes and expands vigor. The paper incorporates the profound neural organization with the well-known NLP models (VADER) to distinguish and remove suppositions inside a given content, joining the stock change close cost and compound score to lessen the speculation hazard. In the event that we accept the information separated from the news for expectation completely, we may expand inclination because of some non-target reports. Accordingly, the DP-LSTM improves the power of the forecast model. [7]

\section{Predicting NASDAQ and NSE Stocks using Machine Learning Algorithms: ARIMA, LSTM \& Linear Regression}

This paper predicts NASDAQ (American) and NSE (Indian) stock costs. The Machine Learning calculations utilized were ARIMA, Linear regression, and Long Short Term Memory. These calculations were applied to the verifiable stock information of the previous 2 years of ongoing stock costs. The stock information was gotten from Yahoo Finance API and Alpha Vantage API for NASDAQ and NSE separately. They derived that ARIMA and LSTM models were more compatible for determining NASDAQ (American Company) stocks. For NSE (Indian Company) stocks, LSTM and Linear Regression demonstrated more productivity.

\section{Prediction of Stock Market using Machine Learning Algorithms}

This paper introduced an examination of AI-supported calculations to assess the stock costs later on to break down market conduct. $\mathrm{R}$ factual programming language has been utilized to make the examination. It gathered that Logisticregression gives exactness in examining market development course and market expectations contrasted with different models like Random Forest, ARIMA, and K-NN. Clarification of these models with their test concentrate on the datasets for various stocks was arranged understanding rate exactness, merits, and demerits. [9]

\section{Social Media Monitoring using Sentiment Analysis of Twitter Data}

The paper examined the expectation of future stock costs utilizing the assumption esteems for each stock. It focuses on two different techniques, Word2vec and N-gram, for deriving insights from sentiments in tweets. Use of sentiment analysis in addition to machine learning algorithms to tweets extracted from Twitter and analyzing the correlation between the stock market movement of company and sentiments in tweets. It proposes a hybrid approach that combines unsupervised learning to cluster the tweets and then performing supervised learning methods for Classification. The creator applied diverse AI methods: Naive Bayes (NB), Maximum entropy, support vector machine (SVM), and so forth, and presumed that NB, SVM with $89.4 \%$ exactness beats different strategies in opinion characterization. It searched for a correlation between Twitter sentiments with stock costs and figured out which words in tweets associate to change in stock cost by doing a postinvestigation of value change and tweets. [10]

\section{Stock Price Prediction Using ARIMA Model}

The creators utilized the ARIMA model to anticipate the stock cost on the information got from New York Stock Exchange (NYSE) and Nigeria Stock Exchange (NSE). They have utilized data sets comprising four features: open, low, close, and high cost. They have accepted the end cost as the objective viewpoint to be anticipated. The explanation for this was that the closing price is the most relevant cost by the day's end. They have exhibited that there is no connection between the autocorrelation capacities (ACFs) and partial autocorrelation capacities (PACFs) utilizing Q-insights and Correlation plots. For the non-fixed information, it was 
made fixed with the assistance of differencing methods. It was reasoned towards the finish of the examination that the ARIMA model is exceptionally helpful for the transient forecast. [11]

\section{A machine learning model for stock market prediction}

In this paper, the proposed model is based on the study of historical data and technical data. It optimizes LS-SVM with a PSO algorithm for daily stock prediction. Levenberg-Marquardt (LM) algorithm is employed as a benchmark for comparison with LS-SVM and LS-SVM-PSO models. This proposed algorithm was tested for many companies belonging to the sector of information \& technology, financials, health care, energy, communications, materials, and industries. Five indicators were calculated from the raw datasets such as Relative Strength Index (RSI), Money Flow Index (MFI), Exponential Moving Average (EMA), Stochastic Oscillator (SO), and Moving Average Convergence/Divergence (MACD). LS-SVM-PSO has a better performance compared to other algorithms like LS-SVM and ANN while the ANN-BP algorithm has the highest error value. [12]

\section{Stock Market Prediction Using Machine Learning}

In this paper, the proposed model is based on the study of historical data and technical data. It streamlines LS-SVM with a PSO calculation for the everyday stock forecasts. LevenbergMarquardt (LM) calculation is utilized as a benchmark for correlation with LS-SVM and LS-SVM-PSO models. This proposed calculation was tried for some, organizations having a place in the area of data and innovation, financials, medical services, energy, communications, materials, and businesses. Five markers were determined from the crude datasets like Relative Strength Index (RSI), Money Flow Index (MFI), Exponential Moving Average (EMA), Stochastic Oscillator (SO), and Moving Average Convergence/Divergence (MACD). LS-SVM-PSO has a superior exhibition contrasted with different algorithms like LS-SVM and ANN while the ANN-BP algorithm has the highest error value. [13]

\section{Predicting Stock Market Trends Using Machine} Learning and Deep Learning Algorithms Via Continuous and Binary Data; A Comparative Analysis

This paper proposed a comparative study of nine machine learning models, namely Random Forest, Decision Tree,
eXtreme Gradient Boosting, Adaptive Boosting, K-Nearest Neighbors, Support Vector Classifier, Naïve Bayes, Artificial Neural Network and Logistic Regression. Along with this Long Short-Term Memory and Recurrent Neural Networks, the deep learning methods were additionally studied. To all these models, a total of ten technical indicators were provided as input values. Two totally different approaches for inputs were inclosed ie. continuous data and binary data. This investigated the effect of reprocessing. The continuous data inputs used the stock trading data such as open price, close price, high value and low value. While the binary data inputs provided a preprocessing step to convert the previous continuous data into the binary form. [14]

\section{NSE Stock Market Prediction Using Deep-Learning Models}

In this paper, the authors have used four types of deep learning architectures which are Recurrent Neural Networks, Multilayer Perceptron, Convolutional Neural Network and Long ShortTerm Memory. All these architectures were used for predicting the future value of the stock of a company from the historical dataset obtained. The results procured were compared with the ARIMA model. Observations were made that the ARIMA model failed to perform higher when put next with the neural networks. [15]

\section{Stock Market Forecasting using Machine Learning: Today and Tomorrow}

In this paper, various stock market prediction techniques such as classification, regression, ensemble, evolutionary, deep learning, hybrid, and ABS have been compared. From the results, Random Forest has the highest accuracy of $80.8 \%$ for the Zagreb Stock Exchange whereas deep learning has the least MAPE value(0.0002) for the Chinese securities index 10(CSI 300). Each model has an edge over the other but also has some limits. These can be because of the datasets used or because of their evaluating factors. Among all, the models which predicted the results with higher precision were fusion or hybrid algorithm. This was because these algorithms incorporate the important features of various individual techniques that they are made up of and the computational time is also enhanced when compared to other techniques. [16]

Table - 1 Comparative Study

\begin{tabular}{|c|l|l|c|l|}
\hline $\begin{array}{c}\text { Sr. } \\
\text { No. }\end{array}$ & \multicolumn{1}{|c|}{ Title } & \multicolumn{1}{|c|}{ Authors } & Year & \multicolumn{1}{|c|}{ Technology Stack } \\
\hline 01. & $\begin{array}{l}\text { Stock Market Prediction using Machine Learning } \\
\text { Algorithms: A Classification Study }\end{array}$ & M. Misra, A. Yadav, H. Kaur & 2018 & $\begin{array}{l}\text { Linear Regression, SVM, } \\
\text { Principle Component } \\
\text { Analysis }\end{array}$ \\
\hline 02. & $\begin{array}{l}\text { Stock Market Prediction Using Machine Learning } \\
\text { Techniques }\end{array}$ & $\begin{array}{l}\text { N. Sirimevan, I. Mamalgaha, } \\
\text { C. Jayasekara, }\end{array}$ & 2019 & $\begin{array}{l}\text { Sentimental Analysis, } \\
\text { Recurrent Neural } \\
\text { Network, LSTM }\end{array}$ \\
\hline 03. & $\begin{array}{l}\text { Survey of stock market prediction using machine } \\
\text { learning approach }\end{array}$ & $\begin{array}{l}\text { A. Sharma, D. Bhuriya, U. } \\
\text { Singh }\end{array}$ & 2017 & $\begin{array}{l}\text { Linear Regression } \\
\text { Algorithm }\end{array}$ \\
\hline 04. & $\begin{array}{l}\text { Stock market prediction using data mining } \\
\text { techniques }\end{array}$ & S. Maini, K. Govinda & 2018 & $\begin{array}{l}\text { SVM and Random } \\
\text { Forest }\end{array}$ \\
\hline
\end{tabular}




\begin{tabular}{|c|c|c|c|c|}
\hline 05 . & $\begin{array}{l}\text { Stock Closing Price Prediction using Machine } \\
\text { Learning Techniques }\end{array}$ & $\begin{array}{l}\text { M. Vijh, D. Chandola, } \\
\text { V. Tikkiwal, A. Kumar }\end{array}$ & 2020 & $\begin{array}{l}\text { Multi-layer Perceptron, } \\
\text { SVM, Least square } \\
\text { classifier }\end{array}$ \\
\hline 06. & $\begin{array}{l}\text { Empirical Study on Stock Market Prediction } \\
\text { Using Machine Learning }\end{array}$ & $\begin{array}{l}\text { R. Sable, S. Goel, } \\
\text { P. Chatterjee }\end{array}$ & 2019 & $\begin{array}{l}\text { K-Nearest } \\
\text { Neighbor,SVM }\end{array}$ \\
\hline 07. & $\begin{array}{l}\text { DP-LSTM: Differential Privacy-inspired LSTM } \\
\text { for Stock Prediction Using Financial News }\end{array}$ & $\begin{array}{l}\text { Xinyi Li1, Yinchuan Li2,1, } \\
\text { Hongyang Yang1, et al }\end{array}$ & 2019 & DP-LSTM , ARMA \\
\hline 08. & $\begin{array}{l}\text { Predicting NASDAQ and NSE Stocks using } \\
\text { Machine Learning Algorithms: ARIMA, LSTM } \\
\text { \& Linear Regression }\end{array}$ & $\begin{array}{l}\text { A. Narote, K. Jadhav , J. Barot, } \\
\text { S. Sawant }\end{array}$ & 2020 & $\begin{array}{l}\text { LSTM, Linear } \\
\text { Regression, ARIMA }\end{array}$ \\
\hline 09. & $\begin{array}{l}\text { Prediction of Stock Market using Machine } \\
\text { Learning Algorithms }\end{array}$ & $\begin{array}{l}\text { Neha Bhardwaj, MD Akil } \\
\text { Ansari }\end{array}$ & 2019 & $\begin{array}{l}\text { Logistic-Regression, K- } \\
\text { NN, Random Forest, } \\
\text { ARIMA }\end{array}$ \\
\hline 10. & $\begin{array}{l}\text { Social Media Monitoring using Sentiment } \\
\text { Analysis of Twitter Data }\end{array}$ & $\begin{array}{l}\text { Rupawari Jadhav, M. S. } \\
\text { Wakode }\end{array}$ & 2017 & $\begin{array}{l}\text { Naive Bayes, Random } \\
\text { Forest, SVM, Maximum } \\
\text { Entropy }\end{array}$ \\
\hline 11. & Stock Price Prediction Using ARIMA Model & $\begin{array}{l}\text { Ayodele A. Adebiyi., Aderemi } \\
\text { O. Adewumi, Charles K. Ayo }\end{array}$ & 2014 & ARIMA \\
\hline 12. & $\begin{array}{l}\text { A machine learning model for stock market } \\
\text { prediction }\end{array}$ & $\begin{array}{l}\text { Osman Hegazy, Omar S. } \\
\text { Soliman and Mustafa Abdul } \\
\text { Salam }\end{array}$ & 2014 & PSO, LS-SVM, \\
\hline 13. & Stock Market Prediction Using Machine Learning & $\begin{array}{l}\text { Ishita Parmar,Ridam } \\
\text { Arora,Sheirsh Saxena, et al }\end{array}$ & 2018 & $\begin{array}{l}\text { LSTM, Regression } \\
\text { Based Model }\end{array}$ \\
\hline 14. & $\begin{array}{l}\text { Predicting Stock Market Trends Using Machine } \\
\text { Learning and Deep Learning Algorithms Via } \\
\text { Continuous and Binary Data; A Comparative } \\
\text { Analysis }\end{array}$ & $\begin{array}{l}\text { M Nabipour, P Nayyeri, } \\
\text { H Jabani, Shahab S, } \\
\text { A Mosavi }\end{array}$ & 2020 & 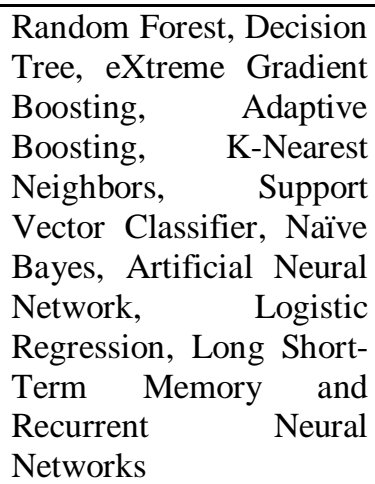 \\
\hline 15. & $\begin{array}{l}\text { NSE Stock Market Prediction Using Deep- } \\
\text { Learning Models }\end{array}$ & $\begin{array}{l}\text { Gopalakrishnan E A, Hiransha } \\
\text { M, Vijay K M, Soman K P }\end{array}$ & 2018 & $\begin{array}{l}\text { Multilayer Perceptron, } \\
\text { Recurrent Neural } \\
\text { Networks, Convolutional } \\
\text { Neural Network, Long } \\
\text { Short-Term Memory }\end{array}$ \\
\hline 16. & $\begin{array}{l}\text { Stock Market Forecasting using Machine } \\
\text { Learning: Today and Tomorrow }\end{array}$ & $\begin{array}{l}\text { Sukhman Singh, Tarun Kumar } \\
\text { Madan, Jitendra Kumar, } \\
\text { Ashutosh Kumar Singh }\end{array}$ & 2019 & $\begin{array}{l}\text { Classification, Deep } \\
\text { learning, hybrid, and } \\
\text { ABS Regression, } \\
\text { Ensemble, Evolutionary }\end{array}$ \\
\hline
\end{tabular}

\section{PROPOSED METHODOLOGY}

In the proposed methodology we will be working with Google Colab for data analysis. We will work with Open, Close, High, Low, Adj. Close and Traded Volume Parameters. The historical data will be collected from Yahoo Finance API.

Selected Algorithms:

1. Linear Regression

Linear Regression is a supervised machine learning algorithm. It is used to predict a correlation amongst more than one independent variable.

$\mathrm{Y} 0=\mathrm{b} 0+\mathrm{b} 1 * \mathrm{x} 1$

Where $\mathrm{Y}=$ Dependent variable
$\mathrm{X}=$ Independent variable

\section{LSTM}

LSTM is an acronym that means Long Short Term Memory networks. This is a special kind of RNN that is capable of learning long-term dependencies. LSTMs are explicitly designed to evade the longterm dependency problem. Remembering information for long periods is peculiarly their default behavior. All recurrent neural networks have the shape of a sequence of repeating modules of neural networks. In standard RNNs, this repeating module will have a really simple structure, like one tanh layer. 


\section{International Journal of Engineering Applied Sciences and Technology, 2021 \\ Vol. 5, Issue 11, ISSN No. 2455-2143, Pages 201-205 \\ Published Online March 2021 in IJEAST (http://www.ijeast.com)}

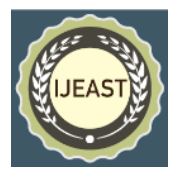

\section{ARIMA}

ARIMA is an acronym that means AutoRegressive Integrated Moving Average, where

AutoRegressive $=$ the model takes the edge of the connection between a predefined number of lagged observations and therefore the present one.

Integrated $=$ differencing between raw observations (eg. subtracting observations at different time steps). Moving Average $=$ the model takes advantage of the connection between the residual error and thus the observations.

It use of three main parameters (p,d,q). These are:

$\mathrm{p}=$ number of lag observations.

$\mathrm{d}=$ the degree of differencing.

$\mathrm{q}=$ the dimensions of the moving average window.

\section{CONCLUSION}

Stock investments are of interest to several investors around the world. However, making a choice may be a complex task as numerous factors are involved. For successful investment, investors are keen to forecast the longer-term situation of the securities market. Even the slightest improvements of predictive efficiency will be very profitable. a top-quality prediction system will help investors make investments more accurate which is more profitable by providing supportive information like the long-run direction of stock prices. This paper discussed and compared the ML algorithms and techniques that are utilized in the stock price prediction. Many studies have proven that the sentiment (mood) analysis features a high impact on future prices. Thus, a mixture of technical and fundamental analyses can produce a highly efficient prediction.

\section{REFERENCES}

[1] Misra, Meghna, et al. "Stock Market Prediction Using Machine Learning Algorithms: A Classification Study." 2018 International Conference on Recent Innovations in Electrical, Electronics and Communication Engineering, ICRIEECE 2018, IEEE, 2018, pp. 2475-78.

[2] Sirimevan, Naadun, et al. "Stock Market Prediction Using Machine Learning Techniques." 2019 International Conference on Advancements in Computing, ICAC 2019, no. 4, 2019, pp. 192-97.

[3] Sharma, Ashish, et al. "Survey of Stock Market Prediction Using Machine Learning Approach." Proceedings of the International Conference on Electronics, Communication and Aerospace Technology, ICECA 2017, vol. 2017-Janua, 2017, pp. 506-09.

[4] Maini, Sahaj Singh, and K. Govinda. "Stock Market Prediction Using Data Mining Techniques." Proceedings of the International Conference on Intelligent Sustainable Systems, ICISS 2017, no. Iciss, IEEE, 2018, pp. 654-61.
[5] Vijh, Mehar, et al. "Stock Closing Price Prediction Using Machine Learning Techniques." Procedia Computer Science, vol. 167, 2020, pp. 599-606.

[6] Sable, Rachna, et al. "Empirical Study on Stock Market Prediction Using Machine Learning." 2019 6th IEEE International Conference on Advances in Computing, Communication and Control, ICAC3 2019, IEEE, 2019, pp. 1-5.

[7] Li, Xinyi, et al. "DP-LSTM: Differential PrivacyInspired LSTM for Stock Prediction Using Financial News." ArXiv, no. NeurIPS, 2019, pp. 1-9.

[8] Narote, Prof Amit, et al. Predicting NASDAQ and NSE Stocks Using Machine Learning Algorithms: ARIMA , LSTM \&. no. Iii, 2020, pp. 39-46.

[9] Bhardwaj, Neha, and Akil Ansari. "Prediction of Stock Market Using Machine Learning Algorithms." International Research Journal of Engineering and Technology, vol. 5994, no. May, 2019, pp. 5994-6005.

[10] Jadhav, Rupawari, and Wakode M. S. "Survey: Sentiment Analysis of Twitter Data for Stock Market Prediction." Ijarcce, vol. 6, no. 3, 2017, pp. 558-62.

[11] Adebiyi, Ayodele A., et al. "Stock Price Prediction Using the ARIMA Model." Proceedings - UKSimAMSS 16th International Conference on Computer Modelling and Simulation, UKSim 2014, 2014, pp. 106-12.

[12] Hegazy, Osman, et al. "A Machine Learning Model for Stock Market Prediction" International Journal of Computer Science and Telecommunications, Volume 4, Issue 12. [Online]. Available: http://arxiv.org/abs/1402.7351

[13] Parmar, Ishita, et al. "Stock Market Prediction Using Machine Learning." ICSCCC 2018 - 1st International Conference on Secure Cyber Computing and Communications, IEEE, 2018, pp. 574-76.

[14] Nabipour, Mojtaba, et al. "Predicting Stock Market Trends Using Machine Learning and Deep Learning Algorithms Via Continuous and Binary Data; A Comparative Analysis." IEEE Access, vol. 8, 2020, pp. 150199-212.

[15] Hiransha, M., et al. "NSE Stock Market Prediction Using Deep-Learning Models." Procedia Computer Science, vol. 132, no. Iccids, Elsevier B.V., 2018, pp. 1351-62.

[16] Singh, Sukhman, et al. "Stock Market Forecasting Using Machine Learning: Today and Tomorrow." 2019 2nd International Conference on Intelligent Computing, Instrumentation and Control Technologies, ICICICT 2019, 2019, pp. 738-45. 